\title{
Aleitamento materno durante a pandemia da COVID-19: Revisão integrativa
}

\author{
Breastfeeding during the COVID-19 pandemic: Integrative review \\ Lactancia materna durante la pandemia de COVID-19: Revisión integradora
}

Recebido: 30/03/2021 | Revisado: 03/04/2021 | Aceito: 06/04/2021 | Publicado: 18/04/2021

Caique Alves Rezende

ORCID: https://orcid.org/0000-0003-0956-7321

Universidade do Estado de Minas Gerais, Brasil E-mail: caiquerezende25@gmail.com

Thays Cristina Pereira Barbosa

ORCID: https://orcid.org/0000-0003-1816-0662

Universidade do Estado de Minas Gerais, Brasil

E-mail: thayscristina19@gmail.com

Isabelle Aparecida de Sousa Bernardes

ORCID: https://orcid.org/0000-0002-5147-2222

Universidade do Estado de Minas Gerais, Brasil

E-mail: isabelleasousa@ hotmail.com

Isabela Teodoro dos Santos

ORCID: https://orcid.org/0000-0001-7839-9486 Universidade do Estado de Minas Gerais, Brasil E-mail: santostisa99@gmail.com

Mariana Rodrigues Moraes Lima

ORCID: https://orcid.org/0000-0003-1382-5725 Universidade do Estado de Minas Gerais, Brasil

E-mail: mariana.moraeslima1@gmail.com

Amanda Aparecida Vieira Camilo ORCID: https://orcid.org/0000-0002-7128-4851 Universidade do Estado de Minas Gerais, Brasil

E-mail: amandavieiracamilo.18@gmail.com

Glória Janaína Rodrigues Bernardo

ORCID: https://orcid.org/0000-0001-5709-9417 Universidade do Estado de Minas Gerais, Brasil

E-mail: janainamorena26@hotmail.com

Maíla Martins Oliveira

ORCID: https://orcid.org/0000-0002-0289-2378 Universidade do Estado de Minas Gerais, Brasil E-mail: maila.oliveira@uemg.br

\begin{abstract}
Resumo
O objetivo deste estudo é compreender como a pandemia do COVID-19 influenciou no aleitamento materno das gestantes nesse período. Refere-se a um estudo de revisão integrativa, realizada em de janeiro de 2021. Assim, o delineamento desse estudo surgiu a partir da seguinte questão de pesquisa: o que se sabe até o momento da literatura científica sobre o aleitamento materno durante a pandemia da COVID-19? Após percorrer as etapas metodológicas foi possível identificar 08 artigos e todas as publicações são datadas no ano 2020 e focadas sobre o aleitamento materno em tempos de pandemia. De acordo com o que foi abordado no presente artigo, o objetivo foi devidamente contemplado demostrando através dos estudos utilizados como base dessa revisão acerca do aleitamento mediante ao cenário da pandemia da COVID-19. Visto isso, torna-se viável continuar os estudos sobre o tema e realizar futuras publicações com novas atualizações do assunto. Pelo fato de ser uma doença na qual ainda há muito que descobrir é essenciais novas produções desse cenário.
\end{abstract}

Palavras-chaves: Aleitamento materno; Infecções por Coronavírus; Pandemias; Recém-nascido.

\section{Abstract}

The aim of this study is to understand how the COVID-19 pandemic influenced the breastfeeding of pregnant women during this period. It refers to an integrative review study, conducted in January 2021. Thus, the outline of this study arose from the following research question: what is known so far in the scientific literature on breastfeeding during the COVID pandemic -19? After going through the methodological steps, it was possible to identify 08 articles and all publications are dated in the year 2020 and focused on breastfeeding in times of pandemic. According to what was discussed in the present article, the objective was duly contemplated, demonstrating through the studies used as the basis for this review about breastfeeding through the pandemic scenario of COVID-19. In view of this, it becomes feasible to continue studies on the topic and to make future publications with new updates on the subject. Due to the fact that it is a disease that there is still much to discover, new productions in this scenario are essential. 
Keywords: Breastfeeding; Coronavirus infections; Pandemics; Newborn.

\section{Resumen}

El objetivo de este estudio es comprender cómo la pandemia de COVID-19 influyó en la lactancia materna de las embarazadas durante este período. Se refiere a un estudio de revisión integradora, realizado en enero de 2021. Así, el esquema de este estudio surgió a partir de la siguiente pregunta de investigación: ¿qué se conoce hasta el momento en la literatura científica sobre la lactancia materna durante la pandemia de COVID -19? Luego de pasar por los pasos metodológicos, fue posible identificar 08 artículos y todas las publicaciones están fechadas en el año 2020 y enfocadas a la lactancia materna en tiempos de pandemia. De acuerdo a lo discutido en el presente artículo, el objetivo fue debidamente contemplado, demostrándose a través de los estudios utilizados como base para esta revisión sobre la lactancia materna a través del escenario pandémico de COVID-19. Ante esto, se hace factible continuar estudios sobre el tema y realizar futuras publicaciones con nuevas actualizaciones sobre el tema. Debido a que es una enfermedad que aún queda mucho por descubrir, las nuevas producciones en este escenario son fundamentales.

Palabras clave: Amamantamiento; Infecciones por coronavirus; Pandemias; Recién nacido.

\section{Introdução}

No dia 11 de março de 2020, a Organização Mundial da Saúde (OMS) declarou que a contaminação pelo novo coronavírus (SARS-COV-2) estava elevada ao grau de pandemia (UNA-SUS, 2020). Com o advento da pandemia da COVID19 no ano de 2020, surgiram muitas incertezas quanto às possibilidades de infecção e os novos cuidados que deveriam ser tomados no que se refere à saúde. Ocorre, então, a produção de pesquisas voltadas principalmente para os grupos de risco. Há, ainda, preocupação com as gestantes, mães e bebês, visto que são mais suscetíveis às enfermidades respiratórias (Dantas, et al., 2020), o que motiva a realização de estudos voltados para esse público durante o contexto pandêmico.

Como é um vírus novo, os estudos estão em desenvolvimento, o que contribui para o aumento das inseguranças quanto ao aleitamento materno, tendo em vista que a maternidade é um fenômeno que já carrega muitas nuances, mudanças fisiológicas e psicológicas, além da reestruturação em âmbito social e cultural. Em meio a esse contexto, surgem dúvidas a respeito dos modos de transmissibilidade e sobre a segurabilidade da amamentação em casos da mãe suspeita ou com COVID19. A principal preocupação é a infecção neonatal, que se contrapõe aos vários benefícios comprovados do leite materno (Tecla, et al., 2020).

Além disso, alguns estudos recomendam o afastamento mãe-bebê pelo período de isolamento (14 dias), mesmo sem evidências da transmissão da SARS-COV-2 através do leite, por precaução, até que a mãe teste negativo para a doença. Todavia, há também posicionamentos a favor da amamentação independentemente disso, visto que os benefícios do leite materno superam os possíveis malefícios caso ocorra à contaminação. A amamentação está diretamente ligada ao desenvolvimento do indivíduo em diversos aspectos, passando pelo o imensurável valor nutricional, a colaboração no desenvolvimento físico das estruturas do rosto, além dos aspectos psicológicos, relacionados ao desenvolvimento da personalidade. (Antunes, et al., 2007). As indicações sobre como deve suceder a amamentação quando há presença de doenças virais nas lactantes são decididas de acordo com a forma de transmissibilidade da doença, além dos riscos e benefícios, isso implica que em alguns casos é recomendado que ela seja mantida e em outros não (Guia Prático De Amamentação -SBP). A discussão e produção de mais estudos perante essa situação se faz necessária, visto que há muitos resultados divergentes entre si quanto à presença ou ausência do vírus no leite materno. Diante da escassez de evidências quanto ao assunto e falta de consenso entre os profissionais de saúde, o tema continua de certo modo nebuloso e carece de mais pesquisas (Tecla, et al., 2020).

Mediante tal cenário, é necessário revisar os estudos já publicados sobre aleitamento materno em tempos de pandemia. Isso se deve às várias implicações do aleitamento ou do afastamento da puérpera em relação ao filho, tal como a vulnerabilidade de gestantes, mães e recém-nascidos a doenças respiratórias (Dantas, et al., 2020); os benefícios a curto e longo prazo da amamentação, bem como prevenção de outras doenças e desenvolvimento imunológico; fortalecimento do 
vínculo mãe-bebê nos primeiros meses de vida e saúde mental da mulher em meio às mudanças e adaptações que sofre nesse período do puerpério (Tecla, et al., 2020).

Esse trabalho justifica-se, então, perante a necessidade de cuidados especiais com um grupo de potencial risco para doenças respiratórias, isto é, puérperas e recém-nascidos, além da escassez de estudos que apresentem conclusões coincidentes entre si quanto ao receio frente à amamentação em casos de mães infectadas ou com suspeita de infecção. Somado a isso, temse a importância do incentivo à amamentação pelos profissionais da saúde, inclusive em tempos de pandemia. O estudo é basilar para a inventividade de estratégias de promoção do aleitamento materno e de ações educativas no que tange o assunto. A revisitação dos artigos e pesquisas realizadas no âmbito do leite materno e infecções por coronavírus é significativo para a delimitação das orientações que devem ser passadas às lactantes, as quais necessitam de direção profissional. O presente estudo teve como objetivo compreender como a pandemia do COVID-19 influenciou no aleitamento materno das gestantes nesse período.

\section{Metodologia}

Refere-se a um estudo de revisão integrativa, realizada a partir de levantamentos bibliográficos em bases de dados acadêmicos, e baseado na experiência vivenciada pelos autores por ocasião da realização de uma revisão integrativa. Foi realizada uma análise qualitativa, através dos resultados encontrados, por meio de abordagem exploratória e interpretação dos dados coletados nas bases (Pereira, Shitsuka, Parreira \& Shitsuka, 2018).

Assim, o delineamento desse estudo surgiu a partir da seguinte questão de pesquisa: o que se sabe até o momento da literatura científica sobre o aleitamento materno durante a pandemia da COVID-19?

Para o levantamento dos artigos na literatura, realizou-se uma busca nas seguintes bases de dados: Scientific Eletronic Library Online (SCIELO), National Library of Medicine National Institutes of Health (PUBMED), Medical Literature Analysis and Retrieval Sistem on-line (MEDLINE), Literatura Latino-Americana e do Caribe em Ciências da Saúde (LILACS), e Base de Dados de Enfermagem (BDENF). A busca foi realizada nas bases de dados em Janeiro de 2021.

A estratégia de busca adotada foi à utilização de descritores e operadores booleanos "OR" e "AND" a fim de aumentar o escopo da busca dos artigos. Na descrição dos descritores foi utilizado o acrômio PICOT, ao qual teve como intuito fortalecer a busca dos artigos nas bases de dados. Foram incluídos descritores e seus termos alternativos, válidos nos Descritores em Ciência da Saúde (DeCS), sendo eles: Aleitamento materno OR Aleitamento OR Alimentação ao Peito OR Amamentação AND Recém-Nascido OR Criança Recém-Nascida OR Crianças Recém-Nascidas OR Lactente Recém-Nascido OR Lactentes Recém-Nascidos OR Neonato OR Neonatos OR Recém-Nascido (RN) OR Recém-Nascidos AND Pandemias OR Pandemia AND Infecções por Coronavirus OR COVID-19.

Foram utilizados os seguintes critérios de inclusão: artigos em português, espanhol ou inglês; artigos originais, disponíveis e gratuitos, que continham um ou mais descritores, artigos publicação no período de 2019 a 2021 e que se enquadravam ao tema abordado. Foram excluídos todos aqueles estudos que não contemplaram o tema a ser abordado. A seleção dos estudos foi realizada por dois pesquisadores, em buscas independentes. Em caso de desacordo, um terceiro autor foi consultado e a decisão final foi tomada por consenso.

A seleção dos artigos foi realizada após a leitura criteriosa dos títulos e resumos para exclusão de publicações duplicadas e daquelas que não atenderem os propósitos da pesquisa. Os artigos selecionados foram lidos na íntegra para extração das variáveis de interesse: Aleitamento materno, pandemia, COVID-19 e recém-nascido.

A análise foi realizada após a leitura na íntegra dos textos. Foi elaborada uma matriz de síntese com os artigos selecionados, contendo as informações: título da obra, autoria, revista e ano de publicação. 


\section{Resultados}

Após a realização da metodologia utilizada referente a revisão integrativa, foi possível identificar 59 artigos, mediante os critérios estabelecidos foram incluídos 8 artigos. Dentre os quais dois se encontravam na base de dados SCIELO e 57 na BVS. Assim sendo, as etapas do processo metodológico foram descritas em um fluxograma (Figura 1).

Figura 1. Etapas do processo metodológico utilizadas para realização do estudo.

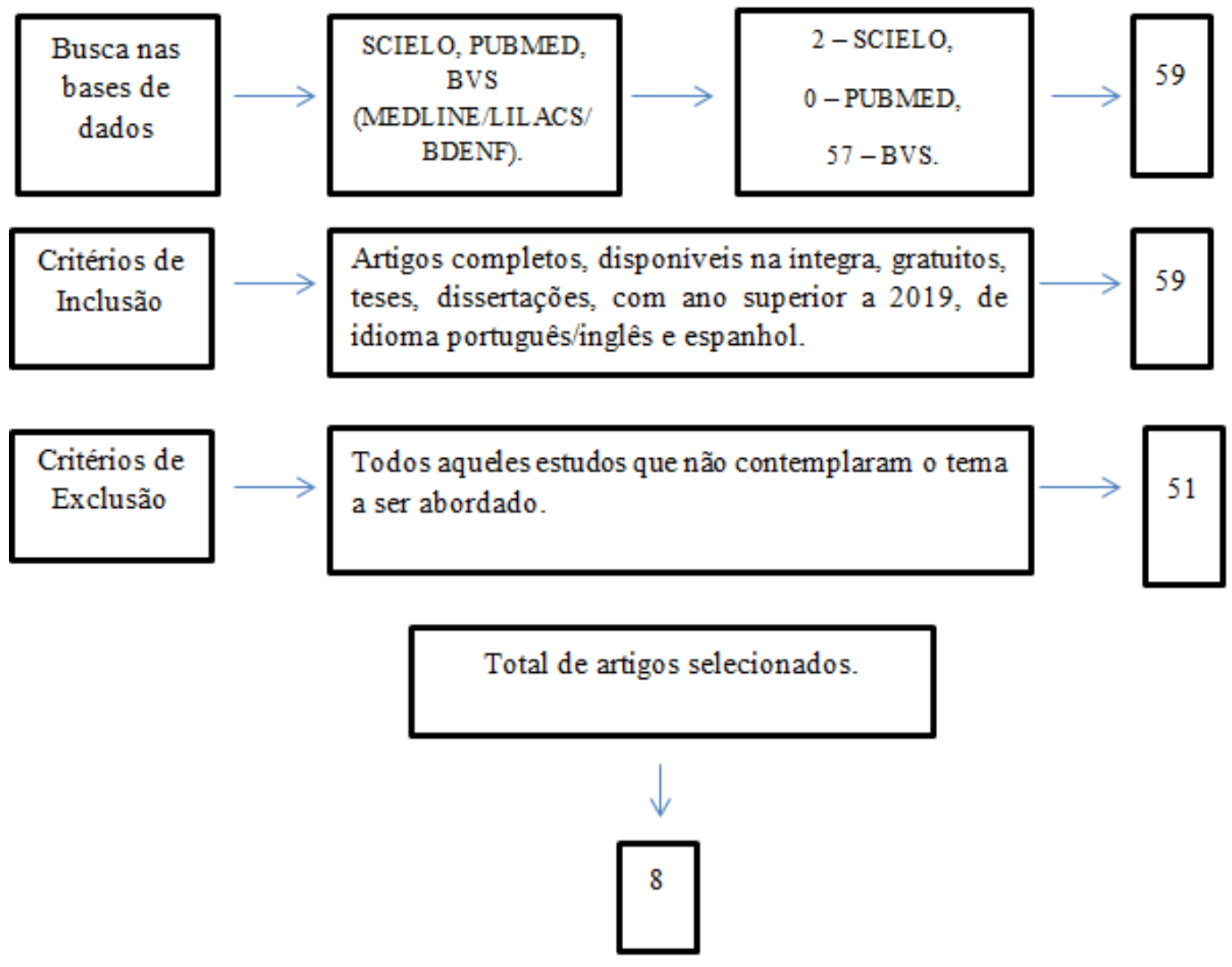

Fonte: Autores (2021).

Após a análise dos estudos encontrados, identificou-se que todos os estudos foram realizados no ano de 2020. Ademais, percebe-se que poucos são os dados referentes a presença de COVID-19 no leite materno (Quadro 1). 
Quadro 1. Distribuição dos artigos utilizados conforme detalhamento quantitativo. Título da obra, autoria, revista e ano.

\begin{tabular}{|c|c|c|c|}
\hline Título da Obra & Autoria & Revista & Ano \\
\hline $\begin{array}{l}\text { To breastfeed or not to breastfeed? Lack of } \\
\text { evidence on the presence of SARS-CoV-2 in } \\
\text { breastmilk of pregnant women with COVID-19 }\end{array}$ & $\begin{array}{l}\text { Paulo Ricardo Martins-FilhoVictor } \\
\text { Santana Santos Hudson P. Santos Jr. }\end{array}$ & $\begin{array}{l}\text { Revista Panamericana de } \\
\text { Salud Pública }\end{array}$ & 2020 \\
\hline $\begin{array}{l}\text { Evaluation for SARS-CoV-2 in Breast Milk From } \\
18 \text { Infected Women. }\end{array}$ & $\begin{array}{l}\text { Christina Chambers, Paul Krogstad, Kerri } \\
\text { Bertrand, Deisy Contreras, Nicole H } \\
\text { Tobin, Lars Bode, Grace Aldrovandi. }\end{array}$ & JAMA & 2020 \\
\hline $\begin{array}{l}\text { Breastfeeding mothers with COVID-19 infection: } \\
\text { a case series. }\end{array}$ & $\begin{array}{l}\text { Augusto Pereira, Sara Cruz Melguizo, } \\
\text { Maria Adrien, Lucia Fuentes, Eugenia } \\
\text { Marin, Azul Forti, Tirso Perez Medina. }\end{array}$ & $\begin{array}{l}\text { Jornal Internacional de } \\
\text { Amamentação }\end{array}$ & 2020 \\
\hline $\begin{array}{l}\text { Negative transmission of sars-cov-2 to hand- } \\
\text { expressed colostrum from sars-cov-2-positive } \\
\text { mothers }\end{array}$ & $\begin{array}{l}\text { Miguel Ángel Marín Gabriel, Ana María } \\
\text { Malalana Martínez, María Eugenia, Marín } \\
\text { Martínez, Jorge Anel Pedroche. }\end{array}$ & Breastfeeding Medicine & 2020 \\
\hline COVID-19 and breastfeeding: what's the risk? & Ivan L. Hand, Lawrence Noble & Journal of Perinatolog & 2020 \\
\hline $\begin{array}{l}\text { Guidance on breastfeeding during the COVID-19 } \\
\text { pandemic. }\end{array}$ & $\begin{array}{l}\text { Valdenise Martins Laurindo Tuma Calil, } \\
\text { Vera Lucia Jornada Krebs, Werther } \\
\text { Brunow de Carvalho. }\end{array}$ & $\begin{array}{l}\text { Revista Da Associação } \\
\text { Médica Brasileira }\end{array}$ & 2020 \\
\hline $\begin{array}{l}\text { Can sars-cov-2-infected women breastfeed after } \\
\text { viral clearance? }\end{array}$ & Guan-jing Lang, Hong Zhao. & $\begin{array}{l}\text { Journal of Zhejiang } \\
\text { University-Science }\end{array}$ & 2020 \\
\hline $\begin{array}{l}\text { The Importance of Continuing Breastfeeding } \\
\text { during Coronavirus Disease-2019: In Support of } \\
\text { the World Health Organization Statement on } \\
\text { Breastfeeding during the Pandemic. }\end{array}$ & $\begin{array}{l}\text { Julianne Williams, Leyla Namazova- } \\
\text { Baranova, Martin Weber, Mehmet Vural, } \\
\text { Julije Mestrovic, Angel Carrasco-Sanz, } \\
\text { Joao Breda, Nino Berdzuli, and Massimo } \\
\text { Pettoello-Mantovani. }\end{array}$ & The Journal of Pediatrics & 2020 \\
\hline
\end{tabular}

Fonte: Autores.

\section{Discussão}

Através da literatura analisada, mediante a pandemia do COVID-19, as mulheres em lactação enfrentaram um grande dilema sobre: “Amamentar ou não?”. Foi possível identificar, que atualmente existem poucos dados relacionados à presença do vírus no leite materno. Assim sendo, encontramos como consequência, o surgimento de diversas dúvidas as lactentes e aos profissionais da saúde sobre a amamentação neste momento que estamos vivenciando. Cabe destacar, que todos vivenciam uma onda de incertezas e almejam que pesquisadores consigam desvendar qual a real indicação ou contraindicação do aleitamento diante do novo coronavírus (Hand \& Noble, 2020).

Após uma vasta leitura, ficou evidente que os dados disponíveis são limitados sobre a presença do COVID-19 no leite materno e a possibilidade de transmissão mãe-bebê (Lang \& Zhao, 2020). As pesquisas realizadas foram feitas a partir da coleta de pequenas amostras de leite e a testagem qRT-PCR, a maioria das amostras coletadas obtiveram resultados negativos. No entanto, evidenciou-se também uma pequena amostra que resultou em positivo para presença do vírus. Houve também, um bebê que testou positivo para COVID-19. Apesar do estudo não existe a certeza de que tenha sido uma transmissão vertical (Hand \& Noble, 2020; Liu W et al., 2020; Pereira et al., 2020).

Dessa forma, atualmente não existem evidências suficientes e concretas que comprovem a transmissão do COVID-19 através de outros fluidos corporais, como no caso de mãe-filho por meio do leite materno (Gabriel, et al., 2020). Visto isso, 
nota-se que o conhecimento científico disponível na atualidade acerca da amamentação por mães infectadas pela COVID-19 é limitado e controverso, sendo necessário evidências consolidadas sobre tal temática (Melo, et al., 2020). Sendo assim, torna-se fundamental a realização de estudos visando avaliar os riscos de longo prazo associados com a interrupção da amamentação e a continuidade da amamentação com mães que apresentam o resultado positivo para a COVID-19.

Destaca-se, que no início da pandemia, surgiram recomendações para que não ocorresse nenhum contato entre o recém-nascido e a mãe, logo, a amamentação não deveria ser realizada, visando evitar a transmissão do COVID-19 para o recém-nascido (Wang SS et al., 2020). Entretanto, em seguida novas recomendações internacionais foram estabelecidas sugerindo os benefícios da amamentação e da relação mãe e filho superando o risco de transmissão (Pereira, et al., 2020).

Por conseguinte, a World Health Organization (WHO, 2020) julga totalmente necessária a amamentação e afirma que os benefícios da mesma excedem o risco de contaminação. Cabe evidenciar, que transmissão pode ocorrer através de gotículas por meio do contato próximo durante a amamentação. Assim, mães com COVID-19 confirmada ou mães sintomáticas com suspeita de COVID-19 devem tomar precauções visando impedir a transmissão ao recém-nascido durante a amamentação (Chaves, Lamounier \& Santiago, 2020). Para isso, a mãe deve seguir alguns cuidados estabelecidos, como: a lavagem das mãos; o uso de máscara durante a amamentação; caso a mulher queira extrair o leite, deve ser feita a higienização da bombinha em cada uso; antes e após o contato com o bebê, limpar e desinfetar minuciosamente todas as superfícies tocadas, entre outros cuidados. Procedimentos que realizados de maneira correta conseguem minimizar a exposição do bebê ao vírus COVID-19 (Calil, et al., 2020; Pereira, et al., 2020).

Infere-se ainda, que no decorrer da pandemia, gestantes e puérperas que foram infectadas com o vírus COVID-19 experimentam medo, anseios e incertezas quanto o cuidado com o recém-nascido (Martins-filho; Santos \& Santos júnior, 2020). Consequentemente, mediante a dúvida sobre a amamentação e os cuidados com o recém-nascido durante a pandemia, é necessário incentivar a tomada de decisões baseadas em evidências científicas com a ajuda e apoio de uma equipe multiprofissional visando os benefícios para a mãe e filho. Com isso, a mãe toma o lugar de fala e terá que optar por amamentar ou não o RN. Dessa maneira, ela deverá ser acompanhada por um profissional da saúde devidamente capacitado que irá apoia-la seja qual for sua decisão (Calil, Krebs \& Carvalho, 2020).

Além disso, podemos identificar também, mulheres que lutam com incertezas e inseguranças e optam pela não amamentação e/ou contato direto com o bebê. Nesses casos, cabe a um profissional capacitado o papel de alimentar o recémnascido, além de ensinar o acompanhante como realizar a extração do leite materno e informar a mãe sobre a periodicidade e importância da amamentação. Se a mulher optar pela não extração do leite materno, pode-se usar os Bancos de Leite Humano. No Brasil, há mais de 220 Bancos de Leite disponíveis para diversas situações, onde o RN não recebe o leite materno (Calil, Krebs \& Carvalho, 2020).

Segundo Calil, et al (2020) mães diagnosticadas com COVID-19 acabam colonizando seus filhos. E, além disso, o leite materno passa a serem potencialmente ricos em anticorpos protetores. Ou seja, os benefícios da amamentação vão além de quaisquer riscos de contaminação vertical. Contudo, os dados presentes na literatura não conseguem afirmar a real possibilidade da transmissão vertical materno-infantil, porém não pode ser descartada. Com isso, todo o cuidado é necessário para preservar saúde da mãe e do RN (Willians, et al., 2020).

Diante de todo conteúdo abordado, torna-se incontestável a incerteza sobre a real possibilidade de contaminação cruzada mãe-bebê por meio da amamentação. Por essa razão, demonstra-se os benefícios do aleitamento materno. Ele protege o RN até a primeira infância, tendo efeito protetor contra diversas doenças infecciosas, além de realizar a transferência de anticorpos e fatores com funções imunológicas para o bebê (WHO, 2020).

Por se tratar de uma revisão de literatura, o estudo limita-se por sua discussão ser baseada no entendimento dos autores após a análise dos estudos encontrados. Além disso a pesquisa foi realizada em um curto período de tempo, podendo 
causar viesses de informações referente as produções publicadas, acredita-se que alguns artigos possam ter sido "excluídos" durante a busca.

\section{Conclusão}

De acordo com o que foi abordado no presente artigo, o objetivo foi devidamente contemplado demostrando através dos estudos utilizados como base dessa revisão acerca do aleitamento mediante ao cenário da pandemia da COVID-19. Ademais ressalta- se a importância de continuar os estudos sobre o tema e realizar futuras publicações com novas atualizações do assunto. Pelo fato de ser uma doença na qual ainda há muito que descobrir é essenciais novas produções desse cenário.

Com as novas visões acerca do tema, as medidas tomadas pelos profissionais da saúde podem se tornar invalidas e sejam necessárias novas medidas para garantir a qualidade do aleitamento. Dessa forma o estudo será de extrema valia para futuras obras desse segmento.

\section{Referências}

Calil, V. M. L. T., Krebs, V. L. J., \& De Carvalho, W. B. (2020). Guidance on breastfeeding during the Covid-19 pandemic. Revista Da Associacao Medica Brasileira, 66(4), 541-546. https://doi.org/10.1590/1806-9282.66.4.541

Chaves, R., Lamounier, J., \& Santiago, L. (2020). Aleitamento materno e terapêutica para a doença coronavírus 2019 (COVID-19). Residência Pediátrica, 10(2), 1-6. https://doi.org/10.25060/residpediatr-2020.v10n2-323

Dantas, A. C., Santos, W. Dos, Nascimento, A. A. D. A., \& Oliveira, L. A. M. de. (2020). Refletindo sobre o contexto da amamentação durante a pandemia do COVID-19. Enfermagem Em Foco, 11(2.ESP), 236-239. https://doi.org/10.21675/2357-707x.2020.v11.n2.esp.3616

Gabriel, M. Á. M., Martínez, A. M. M., Martínez, M. E. M., \& Pedroche, J. A. (2020). Negative Transmission of SARS-CoV-2 to Hand-Expressed Colostrum from SARS-CoV-2-Positive Mothers. Breastfeeding Medicine, 15(8), 492-494. https://doi.org/10.1089/bfm.2020.0183

Hand, I. L., \& Noble, L. (2020). Covid-19 and breastfeeding: what's the risk? Journal of Perinatology, 40(10), 1459-1461. https://doi.org/10.1038/s41372020-0738-6

Joaquim, F. L., Silva, R. M. C. R. A., Garcia-Caro, M. P., Cruz-Quintana, F., \& Pereira, E. R. (2018). Impacto das úlceras venosas na qualidade de vida dos pacientes: revisão integrativa. Revista Brasileira de Enfermagem, 71(4), 2021-2029.

Lang, G. jing, \& Zhao, H. (2020). Can SARS-CoV-2-infected women breastfeed after viral clearance? Journal of Zhejiang University: Science B, 21(5), 405407. https://doi.org/10.1631/jzus.B2000095

Liu, W., Wang, J., Li, W., Zhou, Z., Liu, S., \& Rong, Z. (2020). Clinical characteristics of 19 neonates born to mothers with COVID-19. Front. Med. 14, 193198. https://doi.org/10.1007/s11684-020-0772-y

Martins-Filho, P. R., Santos, V. S., \& Santos, H. P. (2020). To breastfeed or not to breastfeed? Lack of evidence on the presence of SARS-CoV-2 in breastmilk of pregnant women with COVID-19. Revista Panamericana de Salud Publica/Pan American Journal of Public Health, 44, 1-5. https://doi.org/10.26633/RPSP.2020.59

Melo, L. P. C. de, Dias, M. E. da S, Santana, M. S., Diniz, P. R., Galvão, P. V. M., \& Santana, P. de M. S. (2020). Breastfeeding in covid-19 times: an integrative review. Research, Society and Development, 9(9), e129997074. https://doi.org/10.33448/rsd-v9i9.7074

Pereira, A. S., Shitsuka, D. M., Parreira, F. J., \& Shitsuka, R. (2018). Metodologia da pesquisa científica. UFSM.

Pereira, A., Cruz-Melguizo, S., Adrien, M., Fuentes, L., Marin, E., Forti, A., \& Perez-Medina, T. (2020). Breastfeeding mothers with COVID-19 infection: A case series. International Breastfeeding Journal, 15(1), 1-8. https://doi.org/10.1186/s13006-020-00314-8

Rodrigues, S. B., Louro, D., Souza, E. M. O., \& Cunha, M. A. (2020). Estabelecimento da comunicação terapêutica entre enfermeiro e clientes frente á tentativas de autoextermínio: revisão integrativa da literatura. Brazilian Journal of Health Review, 3(3), 5943-5958. https://doi.org/10.34119/bjhrv3n3-152

Tacla, M. T. G. M., Rossetto, E. G., Perdigão, G. M., Zani, E. M., \& Silva, I. V. da. (2020). Reflexões sobre o aleitamento materno em tempos de pandemia por COVID-19. Revista Da Sociedade Brasileira de Enfermeiros Pediatras, 20(spe), 60-76. https://doi.org/10.31508/1676-3793202000000127

UNA-SUS. Organização Mundial de Saúde declara pandemia do novo Coronavírus. (2020). https://www.unasus.gov.br/noticia/organizacao-mundial-de-saudedeclara-pandemia-de-coronavirus

Wang, Ss., Zhou, X., Lin, Xg., Liu Yy., Wu, J.L., Sharifu, L.M., Hu, X.L., Rong, Zh., Luo, W., Chen, Zeng, Wj., Ma, D., Chen, L., \& Feng, L (2020). Experience of Clinical Management for Pregnant Women and Newborns with Novel Coronavirus Pneumonia in Tongji Hospital, China. CURR MED SCI 40, 285-289. https://doi.org/10.1007/s11596-020-2174-4

WHO. World Health Organization. Clinical management of severe acute respiratory infection (SARI) when COVID-19 disease is suspected (2020). https://apps.who.int/iris/bitstream/handle/10665/331446/WHO-2019-nCoV-clinical-2020.4-eng.pdf?sequence=1\&isAllowed=y 
Research, Society and Development, v. 10, n. 4, e46310414475, 2021

(CC BY 4.0) | ISSN 2525-3409 | DOI: http://dx.doi.org/10.33448/rsd-v10i4.14475

Williams, J., Namazova-Baranova, L., Weber, M., Vural, M., Mestrovic, J., Carrasco-Sanz, A., Breda, J., Berdzuli, N., \& Pettoello-Mantovani, M. (2020). The Importance of Continuing Breastfeeding during Coronavirus Disease-2019: In Support of the World Health Organization Statement on Breastfeeding during the Pandemic. Journal of Pediatrics, 223, 234-236. https://doi.org/10.1016/j.jpeds.2020.05.009 